\title{
The 1993 High Energy and Particle Physics Prize
}

\author{
M.J.G. VELTMAN: Massive Yang-Mills Theories
}

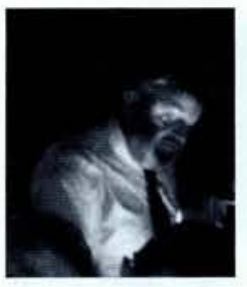

M.J.G. Veltman

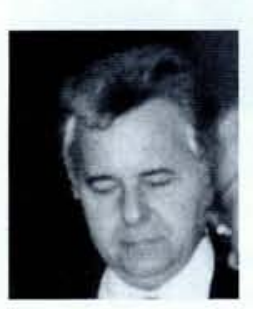

N. Cabibbo

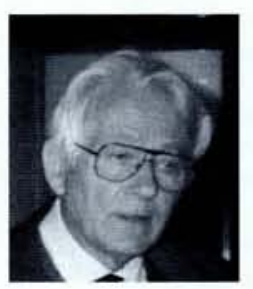

G. Charpak
The 1993 High Energy and Particle Physics Prize of the EPS was awarded to Martinus Veltman, presently professor of physics at The University of Michigan, during the opening ceremony of the 1993 International Europhysics Conference on High Energy Physics (Marseilles, 2228 July) of the High Energy and Particle Physics Division (HEPP) of EPS. It acknowledges "pioneering work on the rôle of massive YangMills theories for weak interactions".

M.J.G. Veltman's work on massive YangMills theories dates back to the years 1960-70, when he was professor at the University of Utrecht and a visitor at CERN and Orsay. Already in 1954, Yang and Mills had shown how to construct a locally gauge-invariant theory based upon the symmetry group SU(2). It was very tempting to extend their work to the description of weak interactions for two reasons. First, Yang-Mills theories naturally describe processes in which a particle decays into another particle with a different charge through the emission of a gauge boson. In weak interaction phenomena, such charge changing events were known to occur frequently. Secondly, YangMills theories have the attractive feature of being renormalizable, i.e., infinities appearing in calculations can be removed through a finite number of redefinitions of physical quantities such as mass and charge. As a result, renormalizable theories have much more predictive power than non-renormalizable ones. Earlier attempts to formulate a theory for weak interactions, which did not incorporate the YangMills ideas, had failed to provide such a renormalizable theory.

The main obstacle for incorporating YangMills symmetry in weak interactions, however, was the fact that the gauge invariance required massless spin-1 fields and that, for the weak interactions, one needed massive fields to describe the short-range nature of the interaction. Unfortunately, a mass term breaks the gauge invariance leading to a non-renormalizable theory.

This did not stop Veltman from investigating the properties of a Yang-Mills theory to which he boldly added a mass term. In particular, he

\section{The High Energy and Particle Physics Prize of EPS}

The HEPP Prize of EPS was awarded to G. Charpak in 1989, to N. Cabibbo in 1991, and to M.J.G. Veltman in 1993. The contributions of these three prizewinners illustrate the dominant themes of the scientific advances in high energy and particle physics in these last 25 years. Charpak's invention of the multiwire proportional chamber was a key step in the electronic revolution which has transformed the collection, on-line analysis, and storage of data from complex multipurpose detectors which so dominate high-energy experiments today. Proportional chambers were important in the experiments which made possible such discoveries as that of the charm in 1974 and of the $\mathrm{W}$ and $\mathrm{Z}$ bosons in 1983.

Cabibbo's "fundamental contribution to the theory of weak interactions leading to the concept of quark mixing" was one of the central ingredients of the Standard Model of particle physics which incorporates both the electroweak theory, and its unification of the $\mathrm{W}$ - and Z-mediated weak interactions with the familiar photon-mediated electromagnetism, together with the QCD of strong interactions.

Finally, Veltman paved the way for a proof of renormalizabilty of the weak interactions and the introduction of a new scalar field, the so-called Higgs particle, which future colliders aim to discover. asked himself the question: what happens with a massive Yang-Mills theory when one lets the mass $M$ of the gauge field go to zero? In other words, does the theory with ever decreasing $M(M \rightarrow 0)$ ultimately coincide with the massless $(M=0)$ theory?

If this were the case, some remarkable cancellations had to occur in the calculations. Indeed, the massive spin-1 propagator, which describes the exchange of this particle, has the form:

$$
\frac{\delta_{\mu v}+k_{\mu} k_{v} / M^{2}}{k^{2}+M^{2}-i \varepsilon}
$$

and the terms $k_{\mu} k_{v} / M^{2}$, which are singular when $M \rightarrow 0$, must somehow be cancelled. Here, $k_{\mu}$ is the four-momentum of the particle.

Veltman's approach to the problem consisted in formally modifying the theory so that new Feynman rules for the perturbation theory emerged, but in such a way that the S-matrix elements, i.e., the physics, remained unchanged. This was achieved by introducing a field redefinition, which nowadays would be called a gauge transformation. One of the results was the introduction of a new effective propagator for the spin-l field given by:

$$
\frac{\delta_{\mu v}-k_{\mu} k_{v} / k^{2}}{k^{2}+M^{2}-i \varepsilon}
$$

with which the $M \rightarrow 0$ limit could be more easily examined.

It thus appeared that massive Yang-Mills theories could be studied in formally different, but physically equivalent formulations. Depending on the questions which were asked, one could now resort to the most suitable formulation. Ultimately, this approach paved the way for a proof of renormalizabilty of the weak interactions: it was given in 1971 by G. 't Hooft in his $\mathrm{Ph}$.D. thesis, for which Veltman was the thesis advisor.

At present, the only known way to generate the masses for the Yang-Mills fields, while preserving the renormalizabilty, is the introduction of a new scalar field, the so-called Higgs field. This new particle has not yet been observed, but, indirectly, it has important implications for the phenomenolgy of the weak interactions. Here too, Veltman made significant contributions leading, among other things, to precise determinations for the masses of the intermediate vector bosons.

Because of his scientific achievements and his pedagogical talents, Veltman became a very popular lecturer at many summer schools. His stubborn belief in the physical significance of Yang-Mills theories, and his persistent analysis of their consequences, contributed greatly to the present success of the Standard Model for weak interactions.

For all this, thank you very much, Tini!

R. Gastmans, Chairman, HEPP Board

The High Energy and Particle' Division gratefully acknowledges donations for the High Energy and Particle Physics Prize from Cray (France), LeCroy (USA), Digital Equipment (Switzerland), Interatom (Germany), Siemens (Germany), Thomson Tubes Electroniques (France), and Philips (The Netherlands). 